Helgoländer wiss. Meeresunters. 17, 257-268 (1968)

\title{
Untersuchungen zur Verträglichkeit von Meer- und Brackwasser für Ciliaten des Saprobiensystems der Wassergütebeurteilung
}

\author{
Hartmut Bick \\ Zoologisches Institut der Universität Bonn, Hydrobiologische Arbeitsgruppe, Bonn
}

\begin{abstract}
Studies on the toleration of sea and brackish water by ciliates of the saprobic system for evaluating water pollution levels. More than $50 \%$ of the indicator organisms of the higher polluted zones are ciliates. Owing to this fact, ciliates are used extensively in laboratory experiments in order to produce more information regarding their ecological tolerances. The present paper reports new results obtained on ciliates exposed to sea and brackish water. The succession of freshwater ciliates associated with the decay of cellulose was investigated in 30-liter basins; detailed information is presented on successions observed in various concentrations of brackish water. When brackish water was used instead of freshwater, the number of ciliate species decreased rapidly, e. g., 40 species were found in freshwater, 26 in $3.5 \% 0 \mathrm{~S}, 10$ in $14 \% \mathrm{~S}$ and 4 in $35 \% \mathrm{~S}$, respectively, Only a few indicator organisms used in fieshwater may, therefore, be of real value as indicators in high concentrations of brackish water. Most of the freshwater ciliates tested are not able to tolerate higher salinities at all.
\end{abstract}

\section{EINLEITUNG}

Bei der biologischen Wassergütebeurteilung von Süßwässern nach dem Saprobiensystem werden zahlreiche Ciliatenarten als Leitformen benutzt, vor allem im polysaproben und alpha-mesosaproben Bereich (Kolkwitz 1950, LieBMANn 1962). Bei der zunehmenden Bedeutung der Wassergüteuntersuchung in Gewässern mit einem gegenüber Süßwasser erhöhten Gesamtsalzgehalt kommt der Frage nach der ökologischen Potenz der Leitformen gegenüber Versalzung im weitesten Sinne erhöhte Bedeutung zu.

Von einer Reihe von Süßwasserciliaten ist bekannt, daß sie mehr oder weniger stark erhöhten Gesamtsalzgehalt ertragen (Literatur siehe Ax \& Ax 1960, Brck 1964a). Eine weitere Uberprüfung des Problemkreises erscheint aber aus folgenden Gründen wichtig: (1) der Anteil der Leitformen an den bisher untersuchten Ciliatenarten ist relativ gering; (2) Untersuchungen zur Populationsdynamik fehlen; (3) die bisher mitgeteilten Salzgehaltstoleranzen differieren erheblich.

Im Rahmen einer umfassenden Bearbeitung der Autökologie der bei der biologischen Selbstreinigung auftretenden Süßwasserciliaten wurde deshalb auch der Ein- 
fluß von erhöhtem Gesamtsalzgehalt auf die Ciliatenbesiedlung von Modellgewässern untersucht. Um eine möglichst umfassende Aussage machen zu können, wurden verschiedene Versalzungstypen überprüf: (1) thalassogenes Brackwasser; (2) athalassogenes Natronseewasser und (3) Versalzung durch Kaliwerksabwässer (vgl. BICK 1964 $a$ und $b, 1967)$. Hier sollen nur einige wesentliche Ergebnisse der Untersuchungen mit thalassogenem Brackwasser referiert werden.

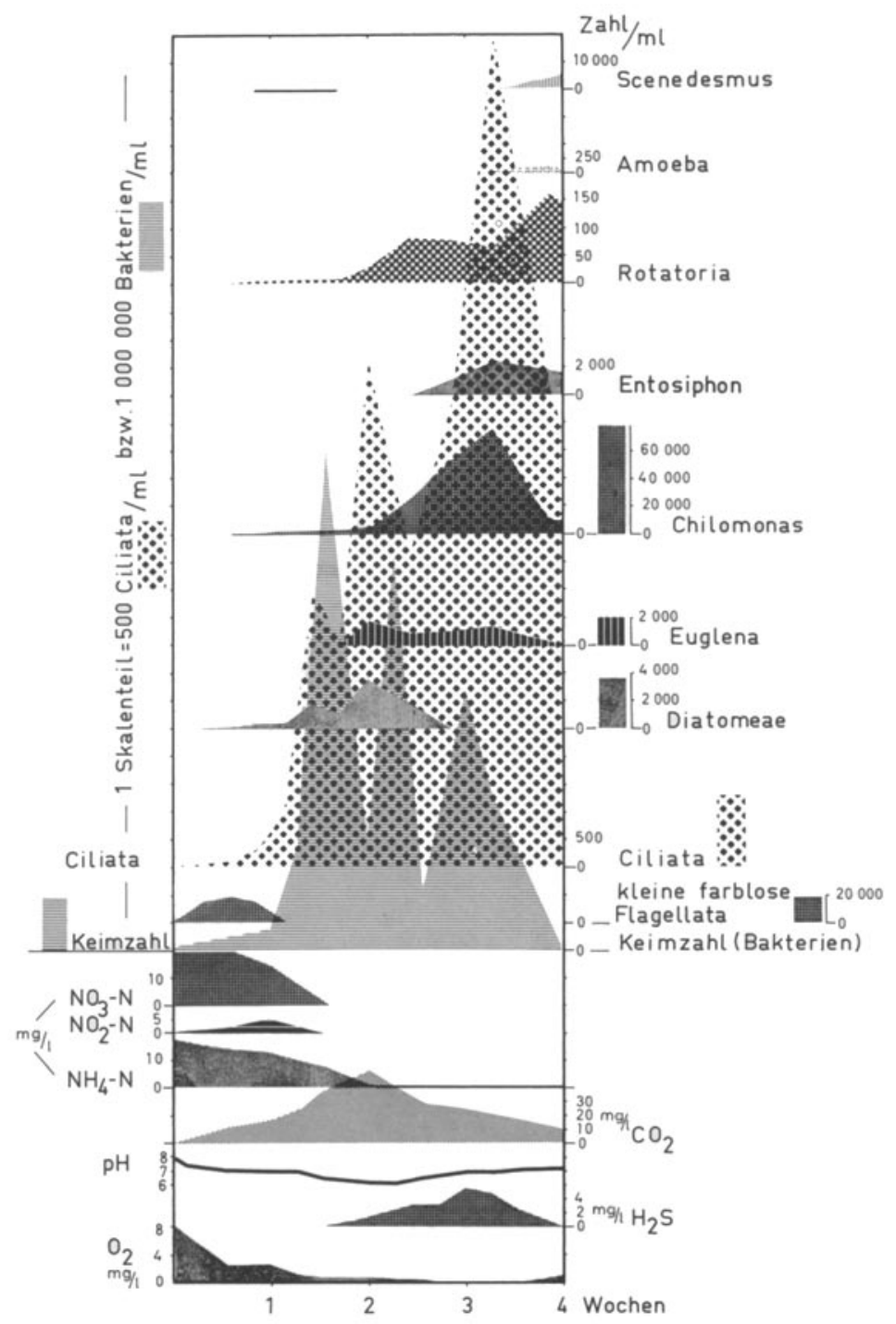

Abb. 1a: Die Veränderungen wichtiger abiotischer Faktoren und die Sukzession der Organismen beim Zellstoffabbau in Süßwasser 


\section{METHODIK}

Bei meinen Untersuchungen wurde folgende Methode benutzt: Modellversuche zur biologischen Selbstreinigung wurden unter standardisierten Bedingungen in 30-Liter-Aquarien angesetzt und die Organismensukzession sowie die abiotischen Ver-

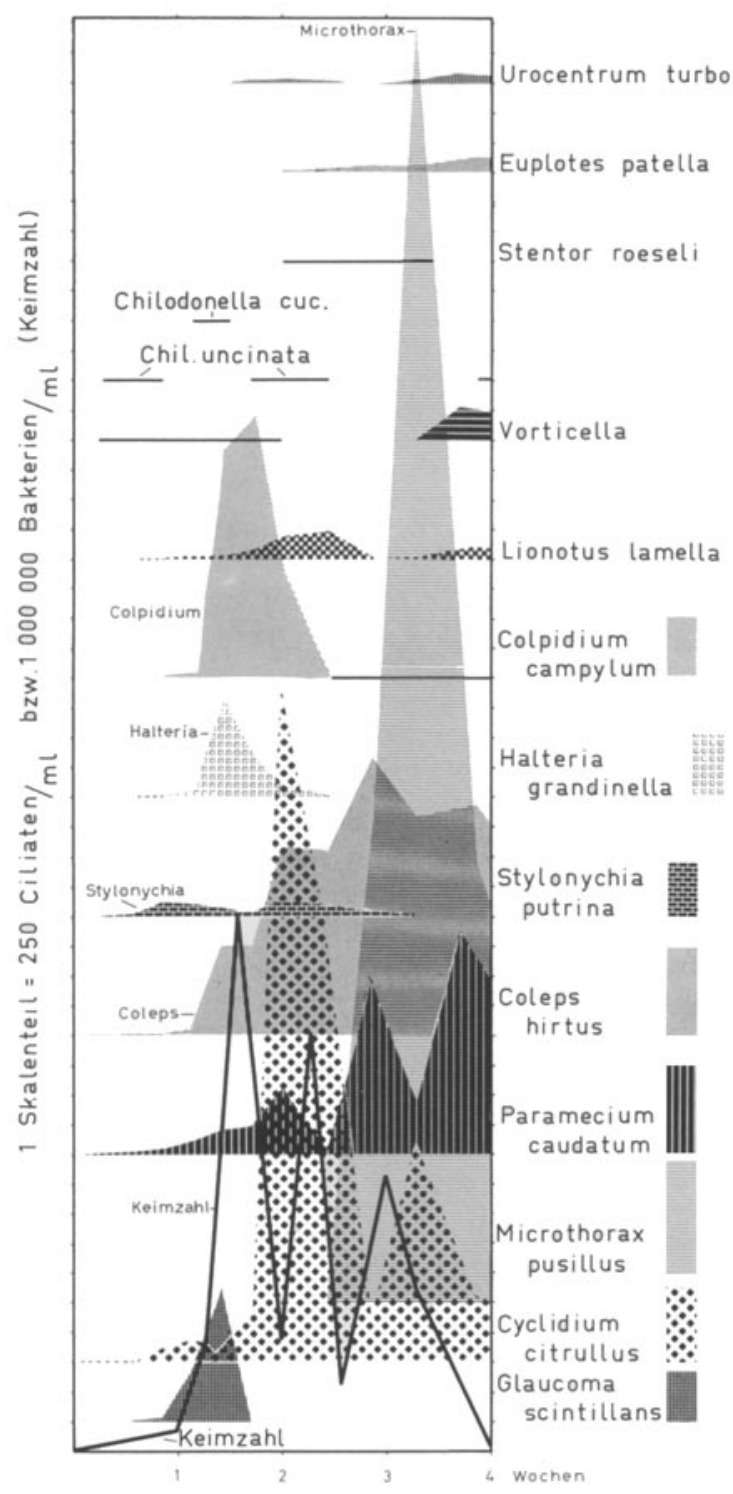

Abb. 1b: Die Sukzession der Ciliaten beim Zellstoffabbau in Süßwasser. Arten mit geringer Individuendichte wurden vernachlässigt, finden sich aber in Tabelle 1 
änderungen im Verlauf des Abbauprozesses verfolgt. Die hier beschriebenen Versuche basieren auf Modellen mit Zellstoffabbau in einem mit anorganischen $\mathrm{N}$ - und $\mathrm{PO}_{4}$ Verbindungen beschickten künstlichen Süß- bzw. Brackwasser. Das Brackwasser wurde nach Lyman \& Fleming (vgl. Remane \& Schlieper 1958) hergestellt. Die Versuchs-temperatur betrug 20 bis $22^{\circ} \mathrm{C}$. Bei einem künstlichen 12-Stunden-Tag belief sich die Versuchsdauer je nach Fragestellung auf 4 bis 16 Wochen. Die Beimpfung erfolgte nur mit Süßwassermaterial (Einzelheiten siehe Bick 1964a).

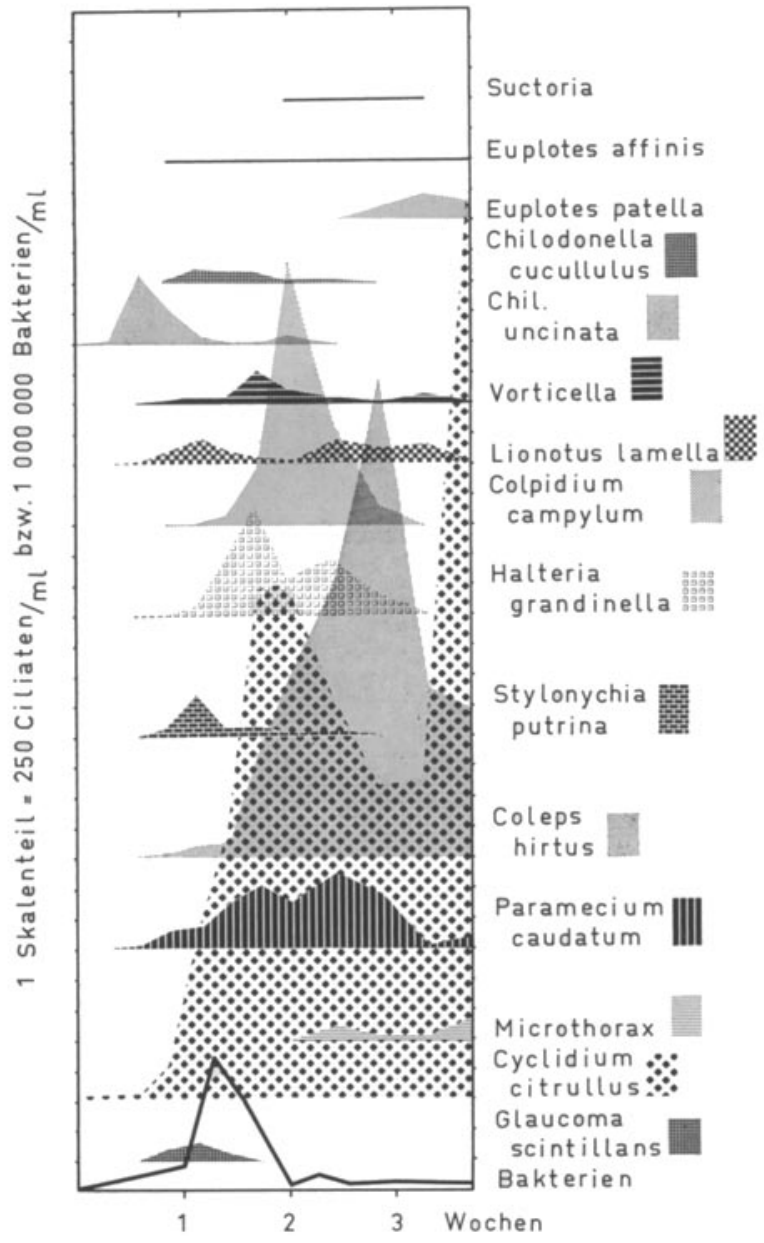

Abb. 2: Keimzahl und Ciliatenbesatz aus einem Zellstoffabbauversuch mit 1,8 \% Gesamtsalzgehalt. Arten mit geringer Individuendichte sind in Tabelle 1 aufgeführt

\section{VERSUCHSERGEBNISSE}

Organismensukzession und Populationsdynamik der Ciliaten sowie allgemeine abiotische Bedingungen in den Modellversuchen sollen zunächst an einem Kontroll- 
versuch mit Süßwasser kurz beschrieben werden (vgl. Abb. 1a und b). Der Zellstoffabbau geht einher mit folgenden kennzeichnenden Milieuveränderungen (Abb. 1a): Der aktuelle $\mathrm{O}_{2}$-Gehalt nimmt in der ersten Woche stark ab, und die 2. bis 3. Woche ist

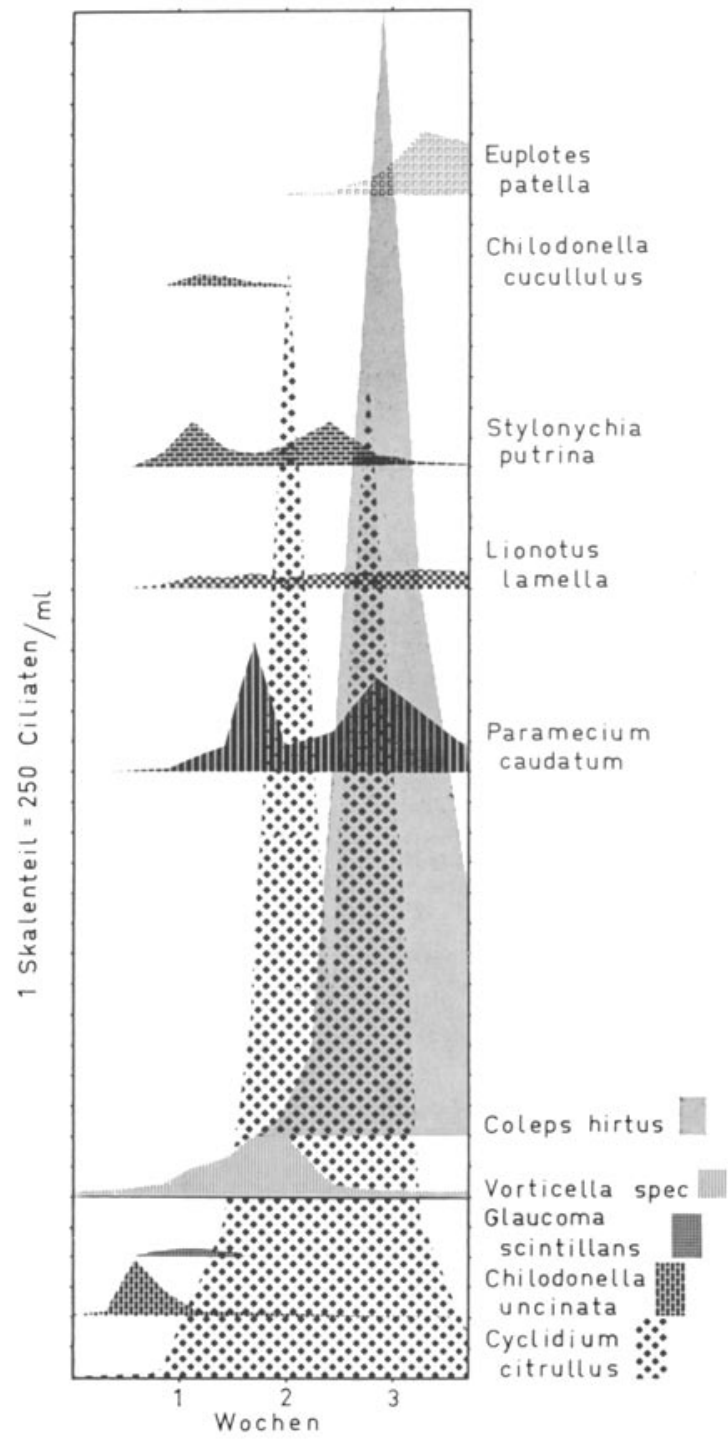

Abb. 3: Die Sukzession der Ciliaten beim Zellstoffabbau in Brackwasser von 3,5\% S. Arten mit geringer Individuendichte sind in Tabelle 1 aufgeführt

durch Sauerstoffmangel und Vorkommen von $\mathrm{H}_{2} \mathrm{~S}$ gekennzeichnet. Der Gehalt an freiem $\mathrm{CO}_{2}$ erreicht am Ende der zweiten Woche ein Maximum, gleichzeitig sinkt der $\mathrm{pH}$-Wert unter den Neutralpunkt. Die bei Versuchsbeginn vorhandenen $\mathrm{NH}_{4}$ - und 
$\mathrm{NO}_{3}$-Verbindungen verschwinden rasch und lassen sich nach der zweiten Woche höchstens noch in Spuren nachweisen.

Die Organismensukzession (Abb. 1a) beginnt mit einem Maximum von kleinen farblosen Flagellaten, denen Bakterien, Ciliaten, Diatomeen, Eugleniden, größere farblose Flagellaten (Chilomonas, Entosipbon), Amöben, Algen und Rotatorien folgen.

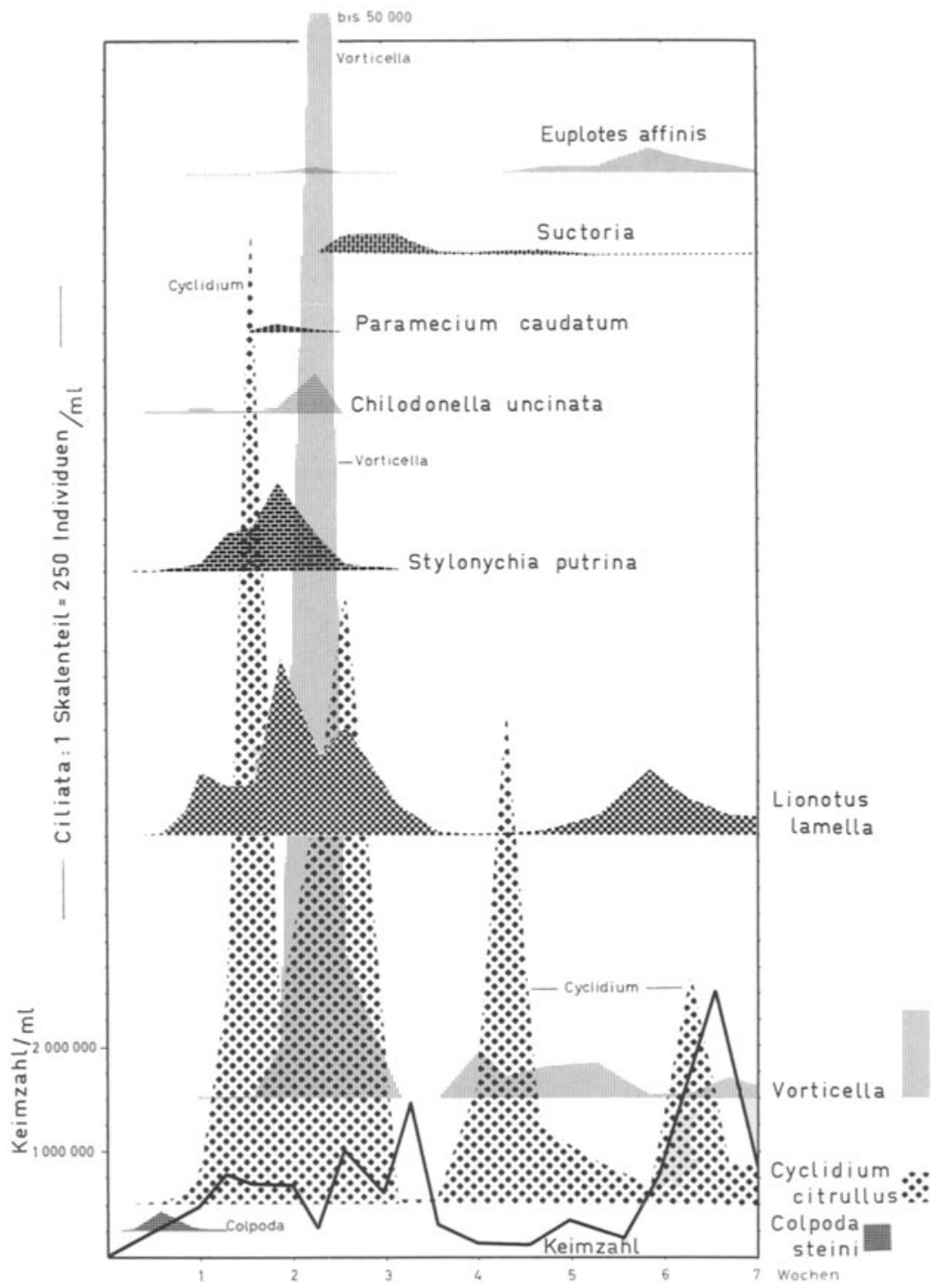

Abb. 4: Die Sukzession der Ciliaten und die Keimzahlkurve aus einem Zellstoffabbauversuch in Brackwasser von $7 \%$ S. Arten, die nur in Einzelstücken auftraten, sind in Tabelle $1 \mathrm{zu}$ finden 
Die hier besonders interessierende Ciliatenbesiedlung wird nach Arten aufgeschlüsselt in Abbildung 1b gezeigt. Erstbesiedler ist Glaucoma scintillans; es folgen starke Bevölkerungsmaxima von Colpidium campylum, Halteria grandinella, Coleps birtus, Stylonychia putrina, Paramecium caudatum, Cyclidium citrullus, Lionotus lamella und Microthorax pusillus. Weitere Arten treten in geringer Bestandsdichte auf (vgl. Tab. 1).

Die eben vorgeführten abiotischen Veränderungen finden sich im Prinzip auch bei den nun zu besprechenden Brackwasserversuchen; Zellstoffabbau erfolgte im ganzen

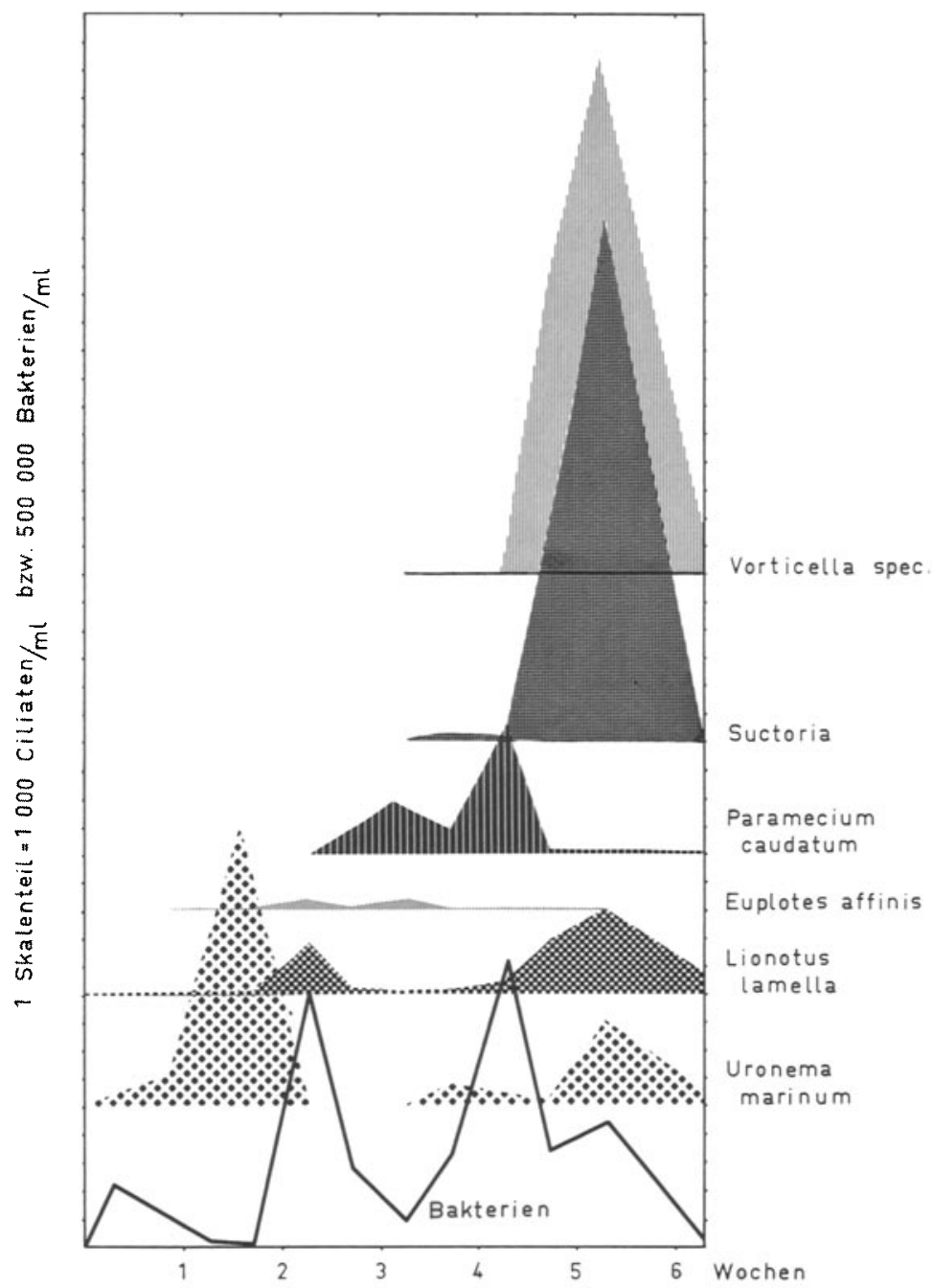

Abb. 5: Keimzahlkurve und Sukzession der Ciliaten in einem Zellstoffabbauversuch mit $10,5 \% \mathrm{~S}$. Einzelfunde sind hier vernachlässigt, finden sich aber in Tabelle 1. (Maßstab verkleinert gegenüber Abbildungen 1 bis 4) 
überprüften Bereich bis zu $35 \%$ S, war aber ab $14 \%$ deutlich verlangsamt. Hieraus ergeben sich gewisse Rückwirkungen auf den Chemismus, wie geringere Sauerstoffzehrung und langsamerer Verbrauch der Stickstoffverbindungen. Die Organismenbesiedlung der Süßwasserkontrollen einerseits und der Brackwasserversuche andererseits zeigt sehr deutliche Unterschiede, die für die Ciliaten im folgenden detailliert dargestellt werden. (Aus einer großen Zahl von Experimenten werden hier nur einige typische herausgegriffen, weitere Beispiele siehe BICK 1964a.)

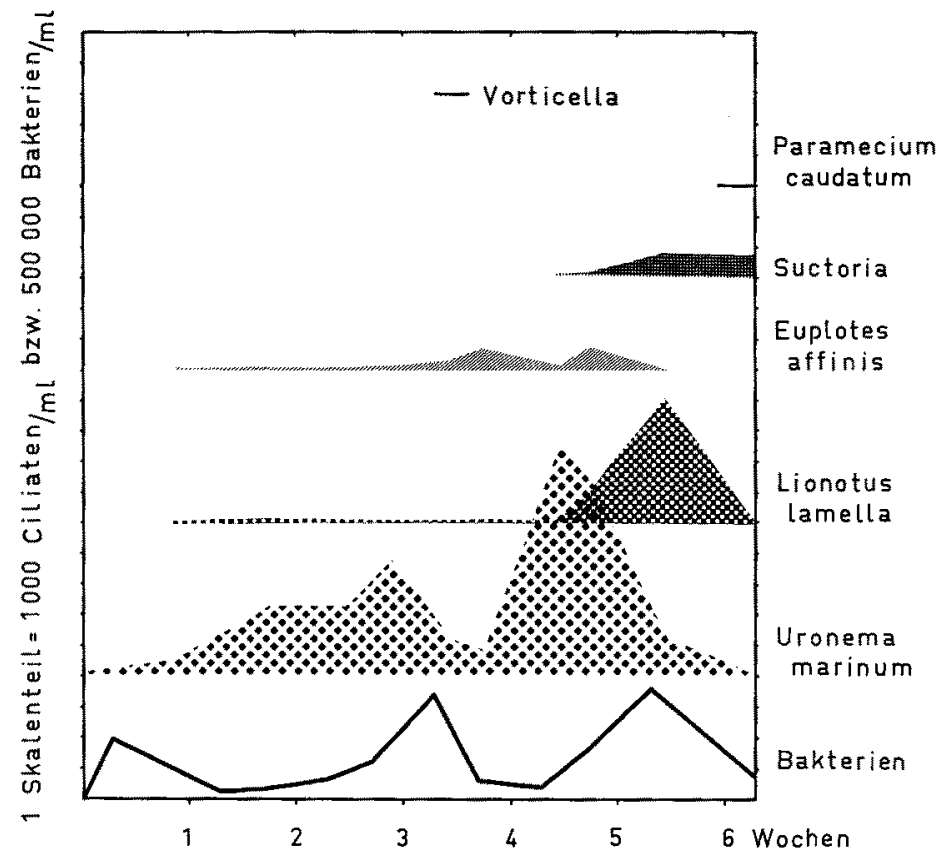

Abb. 6: Keimzahlkurve und Sukzession der Ciliaten in Zellstoffabbauversuch mit $14 \% 0 \mathrm{~S}$. Einzelfunde sind hier vernachlässigt, finden sich aber in Tabelle 1. (Maßstab wie in Abbildung 5 , aber verkleinert gegenüber Abbildungen 1 bis 4)

Eine Erhöhung des Gesamtsalzgehaltes auf 1,8\% $\mathrm{S}$ ergab noch keine wesentliche Veränderung in der Ciliatensukzession (Abb. 2). Ausfälle betrafen nur Arten mit geringer Individuendichte. Glaucoma verliert seine Stellung als Erstbesiedler an Chilodonella uncinata; der Microthorax-Bestand geht zurück.

Bei 3,5\% S setzt sich die angebahnte Entwicklung fort (Abb. 3): Glaucoma verliert weiter an Bedeutung, Chilodonella uncinata bleibt Erstbesiedler. Halteria grandinella und Microtborax pusillus treten nur noch in Einzelstücken auf (vgl. Tab. 1).

Bei $7 \%$ S ist die Artenzahl weiter verringert (Abb. 4 und Tab. 1). Colpoda steini tritt hier als Erstbesiedler auf. Cyclidium und Lionotus sowie Stylonychia putrina sind zahlreich. Auffallend ist die enorme Individuendichte von Vorticella (microstoma-Gruppe), und das starke Auftreten von Suctorien (vor allem Acineta tuberosa) 
und von Euplotes affinis. Die Bestandszunahme dieser letzten drei Gruppen ist kennzeichnend für alle Brackwasserversuche dieser Konzentrationsstufe (vgl. BICK 1964b).

Eine weitere Erhöhung des Gesamtsalzgehaltes auf 10,5\% bringt eine zunehmende Reduktion der Artenzahl mit sich (Abb. 5), die Bestandsdichte der noch existenzfähigen Arten ist jedoch sehr hoch. Wegen der großen Individuenzahl mußte in

Tabelle 1

Übersicht über das Vorkommen der beobachteten Ciliatenarten in den untersuchten Salzkonzentrationen. In den ersten beiden Spalten ist angegeben, welche Arten als Leitformen im Saprobiensystem benutzt werden; K bedeutet die Einstufung nach KoLKwITZ (1950), L nach LIEBMANN (1962). Süßwasser: Kontrolle mit Süßwasser; ps: polysaprob; ams: alphamesosaprob; bms: betamesosaprob; os: oligosaprob

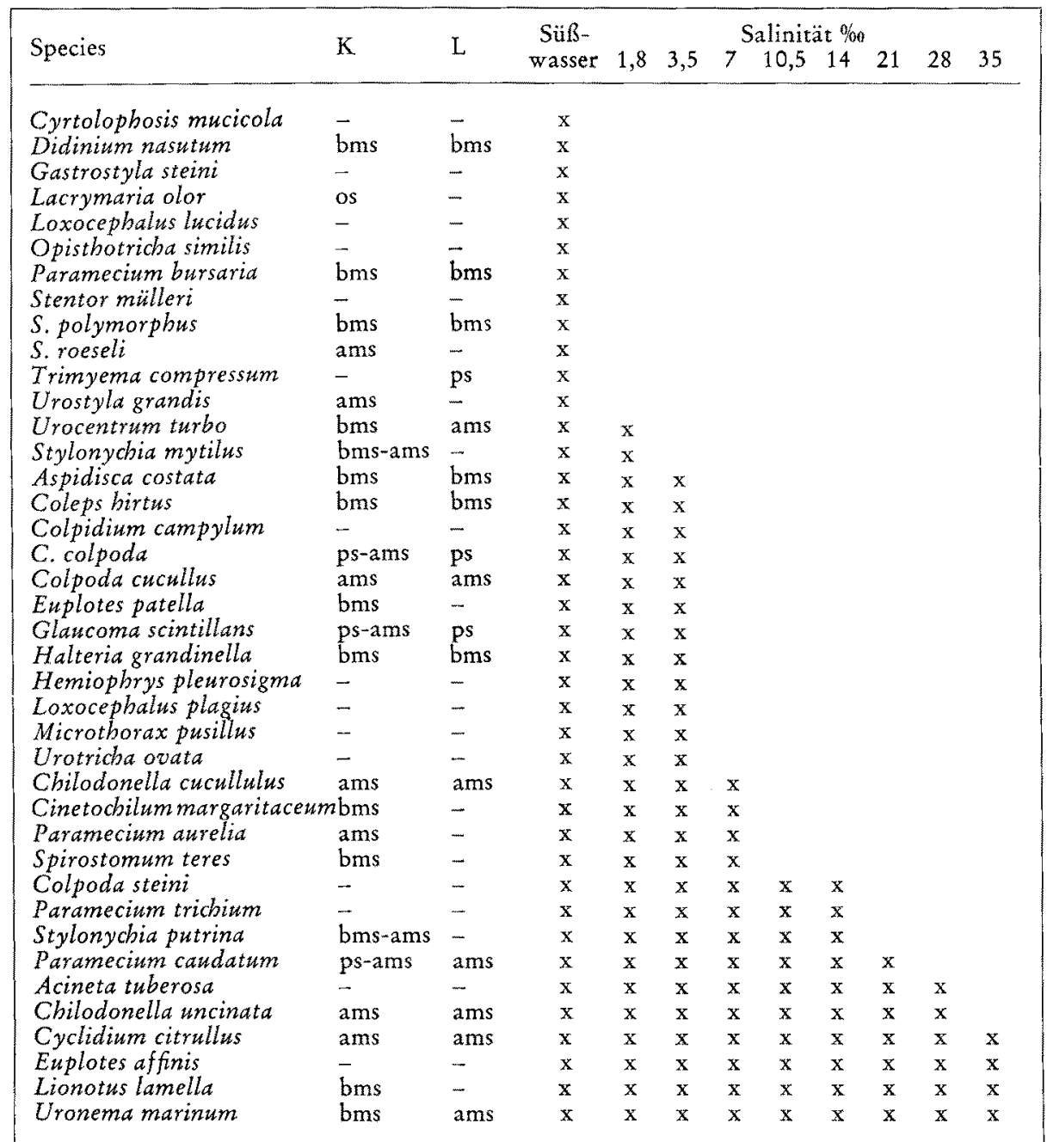


Abbildung 5 der Maßstab gegenüber den vorhergehenden Abbildungen um $1: 4$ verkleinert werden. Auffallend ist hier in Abbildung 5 vor allem der starke Besatz mit Paramecium caudatum und die Massenvermehrung von Suctorien und Vorticelliden.

Von den überprüften noch höheren Brackwasserkonzentrationen soll hier nur noch ein Beispiel gebracht werden. Abbildung 6 zeigt die Ciliatensukzession aus $14 \%$ S; auffällig ist die starke Reduktion der Artenzahl. Die Bestandsdichten hingegen sind bei Uronema marinum, Lionotus lamella, Euplotes affinis und Acineta tuberosa recht hoch.

Die in den höchsten Konzentrationen vorkommenden Ciliatenarten sind in Tabelle 1 zusammengestellt. Hier ist zugleich eine Ubersicht aller beobachteten Arten und ihrer Einstufung ins Saprobiensystem gegeben. Die höchste Toleranz zeigen Cyclidium citrullus, Euplotes affinis, Lionotus lamella und Uronema marinum mit $35 \%$ S. Es folgen Chilodonella uncinata mit $28 \%$ und Paramecium caudatum mit $21 \% \mathrm{~S}$. Die Mehrzahl der untersuchten Süßwasserciliaten fällt also bei relativ geringer Erhöhung des Gesamtsalzgehaltes aus. Einige Formen behalten aber noch im euhalinen Bereich hohe Vitalität und damit eine mögliche Indikatorbedeutung.

\section{DISKUSSION}

In aller Kürze soll noch das eingangs erwähnte Problem erörtert werden, daß die von verschiedenen Autoren gemachten Toleranzangaben zum Teil erhebliche Differenzen zeigen. In diesem Zusammenhang ist bemerkenswert, daß viele Süßwasserciliaten sehr lange Zeit brauchen, um sich an erhöhten Salzgehalt ihres Wohngewässers zu adaptieren. Bei Paramecium caudatum beispielsweise treten sehr lange Verzögerungsphasen im Populationswachstum auf, wenn Süßwassermaterial in Brackwasser überimptt wird. Diese Verzögerungsphase dauerte in einer Versuchsserie bei $7 \%$ S12 Tage, bei $14 \%$ S 6 Wochen und bei $21 \% \mathrm{~S}$ sogar 16 Wochen. Ahnliche Beobachtungen wurden bei Euplotes affinis und Acineta tuberosa gemacht (vgl. Bick 1964a und b). Der Zeitfaktor, d. h. der Zeitraum, der eingeimptten Organismen zur Adaptation zur Verfügung steht, kann demnach große Bedeutung für die Höhe der erzielten Toleranz haben.

Abschließend soll die Frage erörtert werden, ob eine Parallelität besteht $z$ wischen der Einstufung im Saprobiensystem und der Salzverträglichkeit, d.h. ob die sehr eurypotenten polysaproben und alpha-mesosaproben Leitformen auch grundsätzlich

Tabelle 2

Zahl der in verschiedenen Gesamtsalzgehaltsbereichen beobachteten Leitformen der 4 Saprobiestufen (Abkürzungen wie in Tab. 1)

\begin{tabular}{|c|c|c|c|c|}
\hline \multirow[b]{2}{*}{ Salinität } & \multicolumn{4}{|c|}{ Saprobiestufe } \\
\hline & ps & ams & bms & os \\
\hline unter $2 \% 0 \mathrm{~S}$ & 3 & 4 & 4 & 1 \\
\hline 2 bis $4 \% \mathrm{~S}$ & 2 & 3 & 4 & \\
\hline 4 bis $10 \% \mathrm{~S}$ & & 2 & 2 & \\
\hline über $10 \% \mathrm{~S}$ & & 6 & 1 & \\
\hline
\end{tabular}


hohe Resistenz gegen Versalzung zeigen. Gedanken dieser Art äußerte zum Beispiel $\mathrm{K}_{\mathrm{AHL}}$ (1928). Tabelle 2 zeigt die Zahl der in verschiedenen Gesamtsalzgehaltsbereichen beobachteten Leitformen der 4 Saprobie-Stufen. Die polysaproben Formen zeigen keine besonderes auffällige Toleranz, während bei den alpha-mesosaproben Ciliaten ein recht hoher Anteil der untersuchten Arten in erhöhten Salzkonzentrationen vorkommt. Es sei dahingestellt, ob hier eine Parallelität zum Reichtum der alpha-mesosaproben Zone an anorganischen Salzen aus dem Mineralisationsprozeß besteht.

\section{ZUSAMMENFASSUNG}

1. Die Verträglichkeit von Brack- bzw. Meerwasser für saprophile Süßwasserciliaten wurde in Modellversuchen mit Zellstoffabbau untersucht.

2. Repräsentative Sukzessionsdiagramme von ausgewählten Brackwasserkonzentrationen werden mitgeteilt.

3. Die Mehrzahl der geprïften Süßwasserciliaten kam nur im Bereich bis 3,5\% $\mathrm{S}$ vor; mehrere Arten waren aber in der Lage, auch bei hohen Versalzungen zu existieren und erreichten hier sogar hohe Bestandsdichten.

4. Auffallend war, daß bei einigen Arten sehr lange Verzögerungsphasen im Populationswachstum auftraten.

Die Untersuchungen wurden von der Deutschen Forschungsgemeinschaft unterstützt.

\section{ZITIERTE LITERATUR}

Ax, P. \& Ax, R., 1960. Experimentelle Untersuchungen über die Salzgehaltstoleranz von Ciliaten aus dem Brackwasser und Süßwasser. Biol. Z Zbl. 79, 7--31.

Brck, H., 1964a. Die Sukzession der Organismen bei der Selbstreinigung von organisch verunreinigtem Wasser unter verschiedenen Milieubedingungen. Ministerium f. Ernährung, Landwirtschaft und Forsten des Landes Nordrhein-Westfalen, Düsseldorf, $139 \mathrm{pp}$.

- 1964b. Die Populationsdynamik von Süßwasserciliaten in Versuchen mit marinen Brackwässern und binnenländischen Natronwässern. Verh. int. Verein theor. angew. Limnol. 15, 847-852.

- 1967. Vergleichende Untersuchung der Ciliatensukzession beim Abbau von Zellstoff in marinem Brackwasser und athalassogenem Brackwasser vom Typ der Kaliwerksabwässer. Hydrobiologia 29, 185-204.

Kaht, A., 1928. Die Infusorien (Ciliata) der Oldesloer Salzwasserstellen. Arch. Hydrobiol. $19,50-123 ; 189-246$.

Kolkwirz, R., 1950. Okologie der Saprobien. SchrReibe Ver. Wass.-, Boden- u. Lufthyg. 4, $1-64$.

Liebmann, H., 1962. Handbuch der Frischwasser- und Abwasserbiologie. Bd 1. 2. Aufl. Oldenbourg, München, $588 \mathrm{pp}$.

Remane, A. \& Schlieper, C., 1958. Die Biologie des Brackwassers. Schweizerbart, Stuttgart, 348 pp. (Binnengewässer 22.) 


\section{Diskussion im Anschluß an den Vortrag Bick}

RHEINHEIMER: Ich möchte fragen, ob die Arten, die bei Ihren Versuchen die höchsten Salztoleranzen zeigten, in den Brackwasserzonen unserer Flußmündungen regelmäßig vorkommen. Wir mußten nämlich bei unseren bakteriologischen Untersuchungen im Elbe-Astuar und in der Ostsee feststellen, daß ein großer Teil der aus dem Süßwasser isolierten salztoleranten Bakterien im Brackwasser nicht nachzuweisen ist. Diese Formen gedeihen in vitro sehr gut bei Salzgehalten bis 25 oder sogar $40 \%$. Sie scheinen sich aber in der Ostsee nicht entwickeln zu können, sondern gehen dort nach kurzer Zeit zugrunde. Es würde mich nun sehr interessieren, ob das auch bei einigen der salztoleranten Ciliaten der Fall ist.

Brck: Meine Untersuchungen beschränkten sich bisher auf Laboratoriumsexperimente. Vergleichende Freilanduntersuchungen stehen noch aus, so daß ein Vergleich zu den zitierten Verhältnissen bei Bakterien im Augenblick nicht möglich ist.

KINNE: Sie haben ausgeführt, daß einige Populationen sehr lange Zeiten benötigen, um sich an höhere Salzgehalte anzupassen. Wenn ich Sie richtig verstanden habe, betrug die maximale Zeitspanne 16 Wochen. Wie erklären Sie sich diese Anpassung. Denken Sie hierbei primär an eine nicht-genetische Adaptation, an eine Selektion oder an beides?

BICK: Spezielle Untersuchungen zur Frage der Adaptations- oder Selektionsprozesse sind noch nicht angestellt worden. Id möchte daher zunächst auf Erklärungsversuche verzichten.

KINNE: Wie alt werden Einzelindividuen? Mit welchen Generationszeiten muß man unter den Versuchsbedingungen rechnen?

Brcx: Die Generationszahl während der Verzögerungsphase betrug bei dem zitierten 16wöchigen Versuch etwa 10 bis 12.

Heydemann: Bei der Einwanderung von terrestrischen Arthropoden in das Gezeitengebiet der Nordseeküste spielen ökologische Typen der saproben Materialien eine gewisse Rolle. Hier zeigen beispielsweise Dipteren- und Coleopteren-Arten aus saprobem Material des Binnenlands so hohe Salztoleranz, daß sie in erheblicher Artenzahl - beispielsweise in den Salzwiesenbereich des Tidegebiets - einwandern und dort im Detritus leben. In diesem Bereich müssen diese Arten natürlich neben der Salztoleranz auch eine erhebliche Oberflutungstoleranz besitzen. Es liegt vielleicht eine gewisse Parellele zu den von Thnen erwähnten Ciliaten vor. Bei den untersuchten Arthropoden kennen wir neben dem ökologischen Befund des Laborexperiments allerdings auch die existenzökologische Spanne. Klar ist allerdings, daß bei den terrestrischen und semiterrestrischen Arthropoden eine Präadaptation an saline Bereiche wohl nicht in erster Linie über saprobe Milieus erfolgte.

Postma: Vosjan and Srezen of the laboratory in Den Helder studied the salt tolerance of two algae (the fresh water species Scenedesmus and the salt water species Chlamydomonas) by measuring production rates at different salt concentrations. Productivity was measured simultaneously by the $14 \mathrm{C}$ and the oxygen method in a Warburg apparatus. Adaptation time to a specific salinity varied from a few hours to about one day. Productivity decreased with decreasing salinity for Chlamydomonas (to 50\% at 6-8\% S) and with increasing salinity for Scenedesmus (to $50 \%$ at 14-18\% S). Most interesting, the decrease according to the 14-C-method was more rapid than according to the oxygen method, indicating that under stress the oxygen-carbon dioxide ratio changes, or that leakage of organic matters containing $14 \mathrm{C}$ from cells occurs. Possibly, the method of combining the two productivity measurements may also be a promising test to estimate the physiological influence of toxic substances ("Störungsschwelle" according to HaLsBand) on the algae.

BICK: Entsprechende Versuche wurden von mir nicht durchgeführt. 